\title{
On the Monotone Upper Bound Problem
}

\author{
Julian Pfeifle* and Günter M. ZiegleR** \\ TU Berlin, MA 6-2 \\ D-10623 Berlin, Germany \\ \{pfeifle,ziegler\}@math.tu-berlin.de
}

October 15, 2003

\begin{abstract}
The Monotone Upper Bound Problem asks for the maximal number $M(d, n)$ of vertices on a strictly-increasing edge-path on a simple $d$-polytope with $n$ facets. More specifically, it asks whether the upper bound

$$
M(d, n) \leq M_{\mathrm{ubt}}(d, n)
$$

provided by McMullen's (1970) Upper Bound Theorem is tight, where $M_{\mathrm{ubt}}(d, n)$ is the number of vertices of a dual-to-cyclic $d$-polytope with $n$ facets.

It was recently shown that the upper bound $M(d, n) \leq M_{\mathrm{ubt}}(d, n)$ holds with equality for small dimensions $(d \leq 4$ : Pfeifle, 2003) and for small corank $(n \leq d+2$ : Gärtner et al., 2001). Here we prove that it is not tight in general: In dimension $d=6$ a polytope with $n=9$ facets can have $M_{\mathrm{ubt}}(6,9)=30$ vertices, but not more than $27 \leq M(6,9) \leq 29$ vertices can lie on a strictly-increasing edge-path.

The proof involves classification results about neighborly polytopes, Kalai's (1988) concept of abstract objective functions, the Holt-Klee conditions (1998), explicit enumeration, Welzl's (2001) extended Gale diagrams, randomized generation of instances, as well as non-realizability proofs via a version of the Farkas lemma.
\end{abstract}

${ }^{*}$ Supported by the European Graduate Program Combinatorics, Geometry, and Computation (GRK 588/2) in Berlin, the GIF project Combinatorics of Polytopes in Euclidean Spaces (I-624-35.6/1999), and a postdoctoral fellowship at MSRI.

**Partially supported by Deutsche Forschungs-Gemeinschaft (DFG), via the DFG Research Center "Mathematics in the Key Technologies" (FZT86), the Research Group "Algorithms, Structure, Randomness" (Project ZI 475/3), and a Leibniz grant (ZI 475/4) 


\section{Introduction}

In an attempt to understand the worst-case behaviour of the simplex algorithm for linear programming, Motzkin [16] in 1957 considered the maximal number $M_{\mathrm{ubt}}(d, n)$ of facets that a $d$-polytope with $n$ vertices could have, and claimed that the maximum is given by the cyclic $d$-polytopes $C_{d}(n)$ with $n$ vertices; by polarity, $M_{\mathrm{ubt}}(d, n)$ is the maximal number of vertices for a simple $d$-polytope with $n$ facets.

Motivated by the same problem, Klee [1] in 1965 asked for the maximal number $M(d, n)$ of vertices that could lie on a monotone path (that is, an edge-path that is strictly monotone with respect to a linear objective function) on a $d$-polytope with $n$ facets.

Motzkin's claim was substantiated by McMullen [13] in 1970. It seems that traditionally McMullen's result, the Upper Bound Theorem, was also taken as a solution to Klee's question, the Monotone Upper Bound Problem. However, a priori it is only clear that for all $n>d \geq 2$ one has an inequality

$$
M(d, n) \leq M_{\mathrm{ubt}}(d, n)
$$

but it is not at all clear that equality always holds, that is, that for all $n>d \geq 2$ one can construct a simple dual-to-neighborly $d$-polytope with $n$ facets that admits a monotone Hamilton path:

...to answer the original question, we must find out whether both bounds above can be simultaneously tight: It is not clear that for a dual of a cyclic polytope, there can be a monotone path through all, or most, of the vertices. [21. p. 1201]

Thus in [20, Problems $3.11^{*}$ and $8.41^{*}$ ] it was explicitly asked:

The Monotone Upper Bound Problem. How large is $M(d, n)$ ? Does it coincide with $M_{\mathrm{ubt}}(d, n)$ ?

The quest for "bad examples" for the simplex algorithm equipped with specified pivot rules has led to exponential lower bounds for $M(d, n)$. The most prominent one is $M(d, 2 d) \geq 2^{d}$, as seen from the famous Klee-Minty cubes [12]. We refer to Amenta \& Ziegler [2] for a summary of such lower bounds, formulated in the framework of "deformed products." However, these lower bounds are not tight in general: For example, for $d=4$ and $n=8$ the Klee-Minty cubes yield $16 \leq M(4,8) \leq M_{\mathrm{ubt}}(4,8)=20$, while indeed $M(4,8)=20$.

In dimension 5, we have $M(5,7)=12$ according to Gärtner et al. 3]. That is, there is a realization of $C_{5}(7)^{\Delta}$ (which is combinatorially equivalent to a product of a triangle with a tetrahedron) with a monotonely increasing path through all 12 vertices. Moreover, after a projective transformation we may assume that the path is monotone with respect to a linear function $\varphi$ whose unique minimum has value 0 and whose unique maximum has value 1. Thus a deformed prism according to Amenta \& Ziegler [2, Thm. 4.3] will have 24 vertices and a monotone path that visits all of them, proving that $24 \leq M(6,9) \leq M_{\mathrm{ubt}}(6,9)=30$. 
Recently the challenge has been taken up, and it has been proved that the answer to the second question in the Monotone Upper Bound Problem is "YES," that is, $M(d, n)=$ $M_{\mathrm{ubt}}(d, n)$ does hold,

- for small dimensions, $d \leq 4$ (Pfeifle [17, Chapter 5]), and

- for small corank, $n-d \leq 2$ (Gärtner, Solymosi, Tschirschnitz, Valtr \& Welzl [3]).

In the first case, an interesting aspect is that the result cannot be achieved on dual-tocyclic polytopes, but more general dual-to-neighborly polytopes are needed. (These had been missed by Motzkin). The key to the second result is Welzl's concept of "extended Gale diagrams" that will be crucial for our work as well.

In this paper, we give a detailed analysis of some cases of corank $n-d=3$. The main result is that the answer to the Monotone Upper Bound Problem is "NO" in general:

$$
27 \leq M(6,9)<M_{\mathrm{ubt}}(6,9)=30 \text {. }
$$

Our analysis depends on a combination of a number of different techniques and results:

- Any polytope with $M_{\mathrm{ubt}}(d, n)$ vertices is necessarily simplicial dual-to-neighborly. If $n=d+3$ and $d$ is even, then it must be dual-to-cyclic.

- The graphs $G=G\left(C_{d}(n)^{\Delta}\right)$ of dual-to-cyclic polytopes are given by Gale's evenness criterion. For even $d, C_{d}(n)^{\Delta}$ has a dihedral symmetry group of order $2 n$.

- Any linear objective function in general position induces an acyclic orientation on $G$, which is an "abstract objective function" (AOF) as introduced by Kalai, and satisfies the Holt-Klee (HK) conditions. Moreover, in our case it must induce (and be given by) a directed Hamilton path in the graph.

- The symmetry classes of Hamilton paths that induce HK-AOFs are enumerated by computer.

- In terms of Welzl's "extended Gale diagrams" the realizability problem for Hamilton HK AOFs is reformulated as a problem of 3-dimensional Euclidean geometry.

- To prove that some of the Hamilton HK AOFs of interest are indeed realizable we use randomized generation methods.

- To prove non-realizability of AOFs we use a combinatorial technique that may be seen as an oriented matroid version (looking at signs only) of the Farkas lemma; to obtain short proofs, we have implemented automatic search techniques.

Our main findings may be summarized as follows.

Theorem. Let $n=d+3, d \geq 2$. Then a d-polytope with $M_{\mathrm{ubt}}(d, n)$ vertices is necessarily dual-to-neighborly; if $d$ is even, then it is dual-to-cyclic. Hamilton HK AOFs on such polytopes can be classified as follows.

$\boldsymbol{d}=4, \boldsymbol{n}=7$ : There are 7 equivalence classes of Hamilton HK AOFs on the graph of $C_{4}(7)^{\Delta}$; exactly 4 of them are realizable.

In particular, $M(4,7)=M_{\mathrm{ubt}}(4,7)=14$. Moreover, already for $d=4$ and $n=7$ there are non-realizable HK AOFs. (These are the smallest possible parameters.)

$\boldsymbol{d}=\mathbf{5}, \boldsymbol{n}=8$ : There are two types of simplicial dual-to-neighborly polytopes; for the dualto-cyclic one realizable types of Hamilton HK AOFs exist.

In particular, $M(5,8)=M_{\mathrm{ubt}}(5,8)=20$. 
$\boldsymbol{d}=\mathbf{6}, \boldsymbol{n}=\mathbf{9}$ : There are 6 equivalence classes of Hamilton HK AOFs on the graph of $C_{6}(9)^{\Delta}$; none of them are realizable.

In particular, $M(6,9)<M_{\mathrm{ubt}}(6,9)=30$.

\section{The combinatorial model}

If a $d$-polytope with $d+3$ vertices is supposed to have the maximal number $M_{\mathrm{ubt}}(d, d+3)$ of facets then it must be simplicial and neighborly. Thus, by polarity, we are looking at simple dual-to-neighborly $d$-polytopes with $d+3$ facets.

The analysis of such polytopes $P$ is a classical application of Gale diagrams by Perles [6. Sect. 6.2]. It yields that if $d \geq 2$ is even, then the combinatorial type of $P$ is uniquely that of the polar $C_{d}(d+3)^{\Delta}$ of the cyclic $d$-polytope with $d+3$ vertices. For odd $d \geq 3$, more combinatorial types of simple polytopes exist; for $d=3$ as well as for $d=5$ there is exactly one combinatorial type in addition to the dual-to-cyclic polytope (see Altshuler \& McMullen [1]).

The following yields our combinatorial model for the orientations of the graph of $P$ that may be induced by linear objective functions (on some realization of $P$ ).

Definition 2.1. On the graph of a simple $d$-polytope $P$ let $\mathcal{O}$ be an acyclic orientation that has a unique source and sink.

(a) $\mathcal{O}$ is an AOF orientation of $P$ if it has a unique sink in each non-empty face of $P$.

In this case $\mathcal{O}$ also has a unique source in each non-empty face (Kalai [10]; Joswig, Kaibel \& Körner [9]). The orientation is then said to satisfy the AOF condition. Any linear extension of an AOF orientation is called an abstract objective function (AOF) on the vertices of $P$.

(b) $\mathcal{O}$ satisfies the Holt-Klee conditions (or is an HK orientation) if in each $k$-dimensional face of $P$ with $3 \leq k \leq d$ it admits $k$ vertex-disjoint directed paths between the unique source and sink.

(c) $\mathcal{O}$ is an $H K$ AOF orientation if it satisfies (回) and (B) , and a Hamilton HK AOF orientation if it additionally admits a directed Hamilton path from source to sink.

Any linear function in general position (that is, such that no two vertices have the same value) induces an $\mathrm{AOF}$ orientation on the graph of $P$; any such orientation is in fact an HK orientation (Holt and Klee [8]). The negative of the linear function induces the opposite AOF orientation. Any Hamilton AOF orientation induces a unique abstract objective function.

If for some linear function on a $d$-polytope with $d+3$ vertices there is a monotone path through $M_{\mathrm{ubt}}(d, d+3)$ vertices, then the polytope is simple and dual-to-neighborly, and the linear function induces a Hamilton HK AOF. So for our problem we have to enumerate Hamilton HK AOFs on the graphs of dual-to-neighborly $d$-polytopes with $d+3$ facets, which are in fact dual-to-cyclic in the case of even dimension. 


\section{Proposition 2.2.}

(a) The graph of $C_{4}(7)^{\Delta}$ admits exactly 7 equivalence classes (with respect to symmetries of $\mathrm{C}_{4}(7)^{\Delta}$ and global orientation reversal) of Hamilton HK AOFs; they are displayed in Figure 1 .

(b) The polytope $C_{5}(8)^{\Delta}$ admits exactly 1298 equivalence classes of Hamilton HK AOFs.

(c) The polytope $C_{6}(9)^{\Delta}$ admits exactly 6 equivalence classes of Hamilton HK AOFs; they are displayed in Figure 2 .

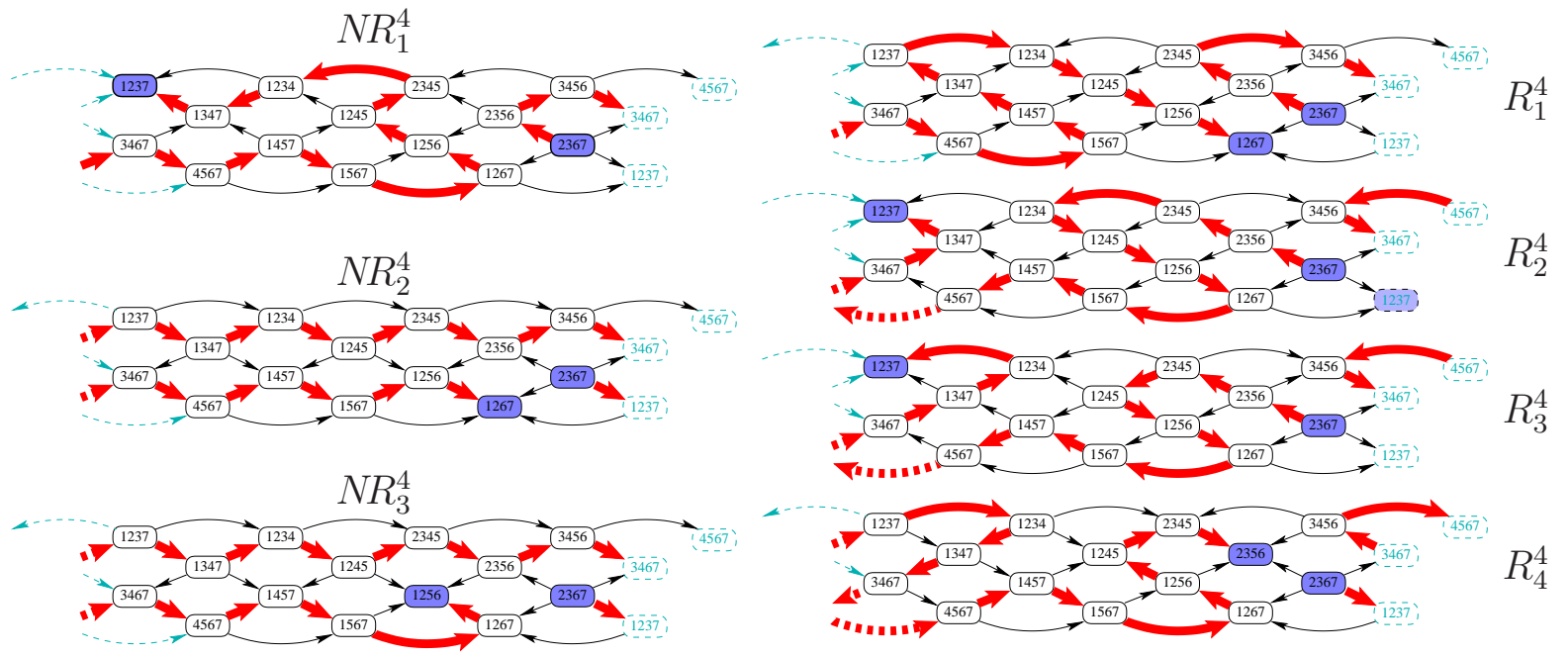

Figure 1: The Hamilton HK AOFs of the graph $G$ of $C_{4}(7)^{\Delta}$. ( $G$ embeds into a Möbius strip [7]). Each vertex is labeled by its set of incident facets, which corresponds to a facet of $C_{4}(7)$. The bold arrows yield the monotone Hamilton paths from source to sink. An arrow $v \rightarrow w$ means that $w$ is higher than $v$; so, for example, $N R_{1}^{4}$ corresponds to

$$
2367<2356<3456<3467<4567<1457<1245<2345<1234<1347<1237 .
$$

Sketch of proof. We enumerate the symmetry classes of directed Hamilton paths in the graph $G$ of one of these polytopes, but prune the search tree whenever the orientation induced by the partial path fails to satisfy the AOF or Holt-Klee conditions.

As an additional pruning criterion, we keep a list $\mathcal{L}_{F}$ of all $\mathrm{HK}$ AOF orientations for each $k$-face $F$ of $P$ for some $3 \leq k \leq \operatorname{dim}(P)$. Whenever we try to add a new oriented edge $e$ to a partial Hamilton path in $G$, we check in all lists $\left\{\mathcal{L}_{F}: e \in F\right\}$ belonging to $k$-faces incident to $e$ whether there still exists an HK AOF orientation containing $e$, and discard all other orientations of that $k$-face.

This strategy was implemented in $\mathrm{C}++$ within the polymake programming environment by Gawrilow \& Joswig [4, 5]; this produced the results listed above.

\section{Extended Gale diagrams}

Welzl's extended Gale diagram [19, 3] encodes the values of a linear objective function on a $d$-dimensional polytope with $n$ facets into an $(n-d)$-dimensional diagram. For this, we 
$N R_{1}^{6}$

$458<258<238<278<478<078<058<038<018<014<012<016<036<034<345<$

$<234<347<147<127<167<678<367<567<056<456<256<236<123<125<145$

$N R_{2}^{6}$ :

$038<238<123<236<234<034<345<347<478<147<014<018<012<016<036<$

$<367<167<678<567<056<256<456<145<458<058<258<125<127<278<078$

$N R_{3}^{6}$ :

$038<238<236<036<016<056<256<567<367<167<678<078<278<478<147<$

$<127<123<012<125<258<058<458<456<145<345<347<234<034<014<018$

$N R_{4}^{6}$ :

$038<238<236<036<016<056<256<567<367<167<678<278<078<478<147<$ $<127<123<012<125<258<058<458<456<145<345<347<234<034<014<018$

$N R_{5}^{6}$ :

$038<058<258<125<256<056<456<458<145<345<034<234<347<147<014<$ $<018<012<016<036<236<367<567<167<678<478<078<278<127<123<238$

$N R_{6}^{6}$ :

$018<058<458<258<125<012<127<278<078<038<238<123<234<034<345<$

$<347<478<147<014<145<456<256<236<036<367<678<567<167<016<056$

Figure 2: Representatives for the six equivalence classes of Hamilton HK AOFs on $C_{6}(9)^{\Delta}$. Each vertex $\boldsymbol{p}$ is given by the 3 -element set $N_{\boldsymbol{p}}$ of the indices of facets not incident to it.

start from a sequence $\left(\boldsymbol{w}_{1}, \boldsymbol{w}_{2}, \ldots, \boldsymbol{w}_{n}, \boldsymbol{g}\right)$ of points in $\mathbb{R}^{d}$ : The $\boldsymbol{w}_{i}$ 's represent the $n$ facetdefining hyperplanes $\left\{\boldsymbol{x} \in \mathbb{R}^{d}: \boldsymbol{w}_{i}^{T} \boldsymbol{x}=1\right\}, i \in\{1,2, \ldots, n\}$, of a full-dimensional polytope $P \subset \mathbb{R}^{d}$ with $\mathbf{0} \in \operatorname{int} P$, and $\boldsymbol{g} \in \mathbb{R}^{d}$ encodes a linear objective function $\boldsymbol{g}^{T} \in\left(\mathbb{R}^{d}\right)^{*}$.

With this interpretation of the input, the extended Gale diagram produces a sequence $\left(\boldsymbol{w}_{1}^{*}, \boldsymbol{w}_{2}^{*}, \ldots, \boldsymbol{w}_{n}^{*}, \tilde{\boldsymbol{g}}^{*}\right)$ of $n+1$ labeled vectors in $\mathbb{R}^{n-d}$ that encodes both the face lattice of $P$ and the orientation $\mathcal{O}_{\boldsymbol{g}}$ of the graph of $P$ induced by $\boldsymbol{g}^{T}$. It is calculated as follows:

(1) Replace $\boldsymbol{g}$ by some positive scalar multiple $\tilde{\boldsymbol{g}}=c \boldsymbol{g}$ such that $\tilde{\boldsymbol{g}}^{T} \boldsymbol{x}<1$ for all $\boldsymbol{x} \in P$; equivalently, $\tilde{\boldsymbol{g}} \in \operatorname{int} P^{\Delta}$.

[This step is optional, and will be modified later. In Welzl's original version of extended Gale diagrams it ensures that the "lifting heights" defined below can be made positive.]

(2) Calculate the standard Gale transform $\left(\boldsymbol{w}_{1}^{*}, \boldsymbol{w}_{2}^{*}, \ldots, \boldsymbol{w}_{n}^{*}, \tilde{\boldsymbol{g}}^{*}\right)$ of the point sequence $\left(\boldsymbol{w}_{1}, \boldsymbol{w}_{2}, \ldots, \boldsymbol{w}_{n}, \tilde{\boldsymbol{g}}\right)$.

Definition 3.1. Let $P=\left\{\boldsymbol{x} \in \mathbb{R}^{d}: \boldsymbol{w}_{i}^{T} \boldsymbol{x} \leq 1,1 \leq i \leq n\right\}$ be a polytope, let $\mathcal{A}_{\boldsymbol{g}}=\left(\boldsymbol{w}_{1}, \boldsymbol{w}_{2}, \ldots, \boldsymbol{w}_{n}, \boldsymbol{g}\right) \subset \mathbb{R}^{d}$ be the sequence of its facet normal vectors, and let $\mathcal{A}_{\boldsymbol{g}}^{*}=\left(\boldsymbol{w}_{1}^{*}, \boldsymbol{w}_{2}^{*}, \ldots, \boldsymbol{w}_{n}^{*}, \tilde{\boldsymbol{g}}^{*}\right) \subset \mathbb{R}^{n-d}$ be the extended Gale diagram of this sequence, whose rows form a basis for the space of affine dependencies among the columns of $\mathcal{A}_{\boldsymbol{g}}$.

For every vertex $\boldsymbol{p}$ of $P$ let $N_{\boldsymbol{p}} \subset\{1,2, \ldots, n\}$ index the $\boldsymbol{w}_{i}$ that correspond to the facets of $P$ that are not incident to $\boldsymbol{p}$. The intersection height $z_{\boldsymbol{p}}$ of $\boldsymbol{p}$ is $z_{\boldsymbol{p}}=-\left(\tilde{\boldsymbol{g}}^{*}\right)^{T} \boldsymbol{z}_{\boldsymbol{p}}$, where $\boldsymbol{z}_{\boldsymbol{p}}=\mathbb{R} \tilde{\boldsymbol{g}}^{*} \cap \operatorname{conv}\left\{\boldsymbol{w}_{i}^{*}: i \in N_{\boldsymbol{p}}\right\}$ is the intersection point of the line $\mathbb{R} \tilde{\boldsymbol{g}}^{*}$ with the convex hull of the $\boldsymbol{w}_{i}^{*}$ s indexed by $N_{\boldsymbol{p}}$. (See Figures 3 and [6]) 
Observation 3.2. After a linear transformation we may assume that $\tilde{\boldsymbol{g}}^{*}=(0,0, \ldots, 0,-1)$. The intersection height $z_{\boldsymbol{p}}$ of a vertex $\boldsymbol{p}$ is then given as the last coordinate of the point where the $(n-d)$-axis meets the affine plane $H_{\boldsymbol{p}}$ through the points $\left\{\boldsymbol{w}_{i}^{*}: i \in N_{\boldsymbol{p}}\right\}$.

Proposition 3.3. Let $\boldsymbol{p}, \boldsymbol{q}$ be vertices of $P$. Then $\boldsymbol{q}$ is higher than $\boldsymbol{p}$ with respect to the linear objective function given by $\boldsymbol{g}$,

$$
\boldsymbol{g}^{T} \boldsymbol{p}<\boldsymbol{g}^{T} \boldsymbol{q}
$$

if and only the intersection height of $\boldsymbol{q}$ is larger than that of $\boldsymbol{p}$,

$$
z_{\boldsymbol{p}}<z_{\boldsymbol{q}}
$$
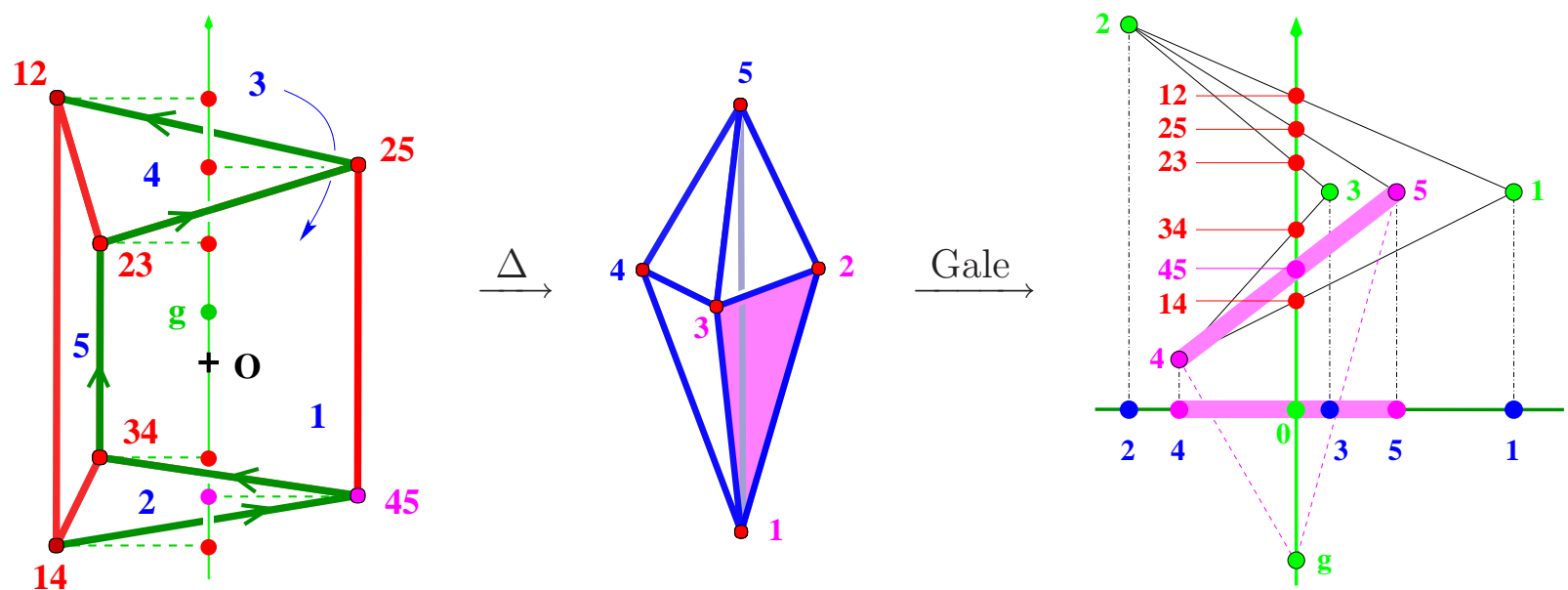

Figure 3: An instance of the extended Gale diagram.

Left: A simple polytope $P$ whose vertices are labeled with the facets they are not incident to, and the ordering $14<45<34<23<25<12$ of the vertices induced by the linear objective function $\tilde{\boldsymbol{g}}^{T}$.

Middle: The simplicial polar polytope $P^{\Delta}$, whose vertices are labeled like the corresponding facets of $P$.

Right: On the base line, a Gale transform of the vertices of $P^{\Delta}$ : Complements of facets of $P^{\Delta}$ correspond to positive circuits (minimal linear dependencies) of (vert $\left.P^{\Delta}\right)^{*}$. Adding $\tilde{\boldsymbol{g}}$ results in a lifting of the Gale transform such that the intersection heights for facet complements of $P^{\Delta}$ encode the ordering of the vertices of $P$ by $\boldsymbol{g}^{T}$.

Example 3.4. Let $P$ be a triangular prism in $\mathbb{R}^{3}$ (see Figure 3 left) with $n=5$ facets. The polar $P^{\Delta}$ is the polytope of Figure 3 (middle) with 5 vertices, and the Gale transform of $P^{\Delta}$ consists of 5 points in $\mathbb{R}^{5-3-1}=\mathbb{R}^{1}$ (Figure 3 right, base line). We obtain the extended Gale diagram in $\mathbb{R}^{2}$ by additionally encoding a linear objective function via a level hyperplane that does not intersect $P$, which corresponds to a point in the relative interior of $P^{\Delta}$. Proposition 3.3 says that in the extended Gale diagram, the value $\boldsymbol{g}^{T} \boldsymbol{p}$ of the objective function is encoded by the height of the intersection of $\mathbb{R} \tilde{\boldsymbol{g}}^{*}$ with the triangle spanned by the points $\boldsymbol{w}_{i}^{*}$ that correspond to facets of $P$ that do not contain $\boldsymbol{p}$. 


\section{Finding realizations}

\section{Proposition 4.1.}

(a) The equivalence classes $R_{1}^{4}-R_{4}^{4}$ of Hamilton HK AOF orientations of the graph of $C_{4}(7)^{\Delta}$ (as given by Figure 11) are realizable. In particular, $M(4,7)=M_{\mathrm{ubt}}(4,7)=14$.

(b) There exist realizable Hamilton $H K$ AOF orientations of the graph of $C_{5}(8)^{\Delta}$. In particular, $M(5,8)=M_{\mathrm{ubt}}(5,8)=20$.

(c) There exist realizations of $C_{6}(9)^{\Delta}$ with 27 vertices on a monotone path.

Sketch of proof. The realizations in (a) and (b) were found by the following procedure. For each polytope $P=C_{d}(d+3)^{\Delta}$, randomly generate a Gale transform $\mathcal{G}\left(P^{\Delta}\right)=$ $\left(\boldsymbol{v}_{1}^{*}, \boldsymbol{v}_{2}^{*}, \ldots, \boldsymbol{v}_{d+3}^{*}\right)$ of $P^{\Delta}$, and for each vertex $\boldsymbol{p}$ of $P$ express the intersection height $z_{\boldsymbol{p}}$ as a linear function of the lifting heights $h_{i}$, where $\left(\boldsymbol{w}_{i}^{*}=\left(\boldsymbol{v}_{i}^{*}, h_{i}\right): 1 \leq i \leq d+3\right)$ is an extended Gale transform of $P$. Now check whether the linear program

$$
z_{\boldsymbol{p}}-z_{\boldsymbol{q}} \leq-1 \quad \text { for all oriented edges } e=(\boldsymbol{p}, \boldsymbol{q}) \text { in } \mathcal{O}
$$

in the variables $h_{1}, h_{2}, \ldots, h_{d+3}$ is feasible, for $\mathcal{O}$ one of the Hamilton HK AOF orientations of $P$. If so, the polar dual of the Gale transform of $\mathcal{G}\left(P^{\Delta}\right)$ yields a realization of the combinatorial type of $P$, and the lifting heights solving the linear program yield a linear objective function that induces the orientation $\mathcal{O}$ on this realization. If not, repeat.

The realizations in (c) were found by lifting randomly generated instances $\left\{v_{1}^{*}, v_{2}^{*}, \ldots, v_{9}^{*}\right\}$ of Gale diagrams of $C_{6}(9)$ to the rational surface $h=\prod_{i=1}^{9} 1 / \ell_{i}(\boldsymbol{x})$, where $\ell_{i}(\boldsymbol{x})$ is the equation of the line $\mathbb{R}\left(v_{i}^{*}-v_{i+1}^{*}\right)$ for $1 \leq i \leq 9$, where $v_{10}^{*}:=v_{1}^{*}$.

Remark. Following a suggestion of Walter Morris, we also searched for long ascending paths on the product $P=\Delta^{2} \times \Delta^{2} \times \Delta^{2}$ of three triangles. This is a simple 6 -dimensional polytope with 9 facets, 27 vertices, and 81 edges. Its graph admits many Hamilton paths that induce HK AOF orientations (800928 in total, that is, 4944 of them beginning in a given directed edge), but we have not been able to find realizations of $P$ with more than 25 vertices on an ascending path.

\section{Proving non-realizability}

Our strategy for proving non-realizability of orientations may be summarized as follows. For each candidate orientation $\mathcal{O}$ of the graph of a polytope $P$ (of even dimension $d$, with $d+3$ facets), we assume that there is a realization of $P$ and a linear objective function $\boldsymbol{g}^{T}$ that induces $\mathcal{O}$ on $P$ 's graph. Each oriented edge of $\mathcal{O}$ then imposes a linear inequality on the lifting heights of the extended Gale diagram of $\left(P, \boldsymbol{g}^{T}\right)$. For some orientations $\mathcal{O}$, a combinatorial version of the Farkas Lemma implies that these inequalities are inconsistent, thereby proving the non-realizability of $\mathcal{O}$. 


\subsection{Inequalities induced by edges}

We start with some notation for vector configurations in $\mathbb{R}^{2}$ and $\mathbb{R}^{3}$. The shorthand $[d+3]$ will denote $\{1,2, \ldots, d+3\}$.

Convention 5.1. For $i \in[d+3]$, we write $\boldsymbol{i}$ for a vector $\left(x_{i}, y_{i}\right)^{T} \in \mathbb{R}^{2}$, and $\boldsymbol{i}^{\perp}$ for the vector $\left(y_{i},-x_{i}\right)^{T}$ orthogonal to $\boldsymbol{i}$ that is obtained by rotating $\boldsymbol{i}$ in the clockwise direction. With this convention, the following relations hold for scalar products:

$$
\boldsymbol{i} \boldsymbol{j}^{\perp}=x_{i} y_{j}-x_{j} y_{i}=\operatorname{det}(\boldsymbol{i}, \boldsymbol{j})=-\operatorname{det}(\boldsymbol{j}, \boldsymbol{i})=-\boldsymbol{j} \boldsymbol{i}^{\perp}=-\boldsymbol{i}^{\perp} \boldsymbol{j} .
$$

We further abbreviate

$$
i j^{\perp}:=\operatorname{sign}\left(\boldsymbol{i} \boldsymbol{j}^{\perp}\right), \quad[\boldsymbol{i} \boldsymbol{j} \boldsymbol{k}]:=\operatorname{det}\left(\begin{array}{ccc}
\boldsymbol{i} & \boldsymbol{j} & \boldsymbol{k} \\
1 & 1 & 1
\end{array}\right), \quad[i j k]:=\operatorname{sign}([\boldsymbol{i} \boldsymbol{j} \boldsymbol{k}]) .
$$

\section{Lemma 5.2.}

(a) If $\boldsymbol{i}, \boldsymbol{i}+\boldsymbol{j}, \boldsymbol{j} \in \mathbb{R}^{2}$ come in anti-clockwise order around 0 , then $i j^{\perp}=+$.

(b) If in a configuration of four vectors $\boldsymbol{i}, \boldsymbol{j}, \boldsymbol{k}, \boldsymbol{\ell} \in \mathbb{R}^{2} \backslash\{0\}$ the vectors $\boldsymbol{i}, \boldsymbol{j}, \boldsymbol{k}$ are ordered clockwise around $0, \boldsymbol{j} \in \operatorname{relint} \operatorname{cone}(\boldsymbol{i}, \boldsymbol{k}),[i j k]=+$, and $\in \operatorname{relint} \operatorname{cone}(-\boldsymbol{i},-\boldsymbol{k})$, then $[i \ell j]=[j \ell k]=+$.
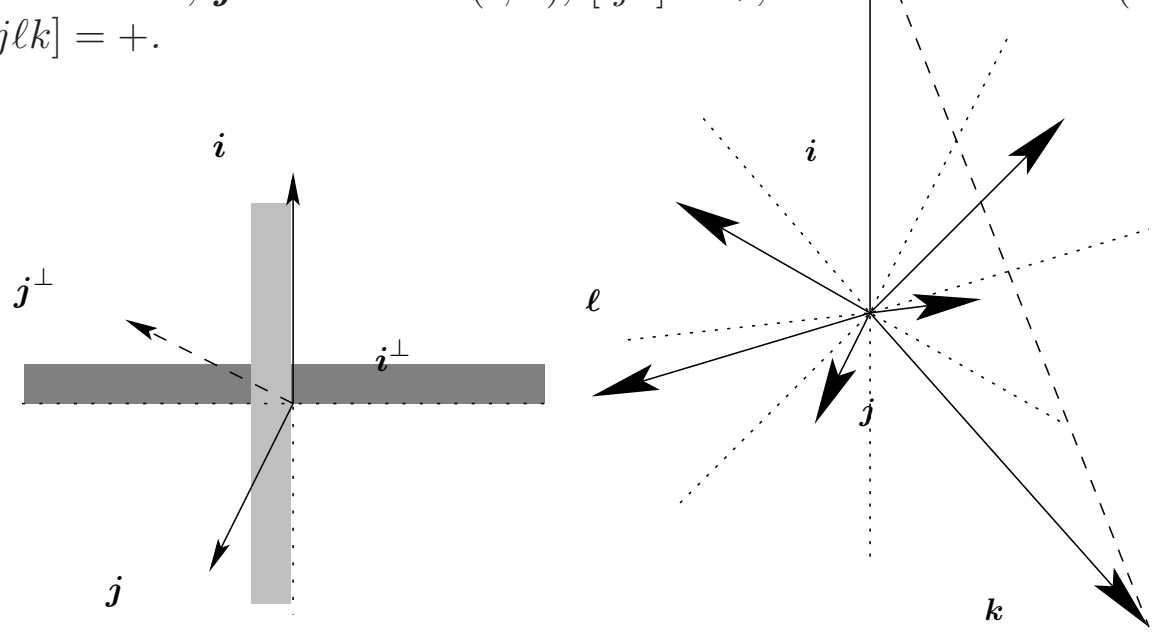

FiguRE 4: Deducing sign patterns.

Left: If $\boldsymbol{i}, \boldsymbol{i}+\boldsymbol{j}, \boldsymbol{j} \in \mathbb{R}^{2}$ come in anti-clockwise order around 0 , then $i j^{\perp}=+$.

Right: In this situation, if $[i j k]=+$, then $[i \ell j]=[j \ell k]=+$.

Convention 5.3. The vertices of $C_{d}(d+3)$ are labeled by $[d+3]$ in the natural order, so that the facets are given by certain $d$-subsets of $[d+3]$ according to Gale's evenness criterion. The vectors in any Gale transform are then labeled so that $\mathbf{1}, \mathbf{3}, \mathbf{5}, \mathbf{7}, \ldots, \mathbf{2}, \mathbf{4}, \mathbf{6}, \ldots$ come in clockwise order around the origin. We identify each facet of $C_{d}(d+3)$ with the indices of the three vertices it misses, so that ordering this index set yields a triangle with anti-clockwise orientation that encloses the origin (cf. Figure 5 ).

Now we polarize. Correspondingly, we label each vertex $\boldsymbol{p}$ of $C_{d}(d+3)^{\Delta}$ by the 3-element set $N_{\boldsymbol{p}}$ of (indices of) the facets it does not lie on. 


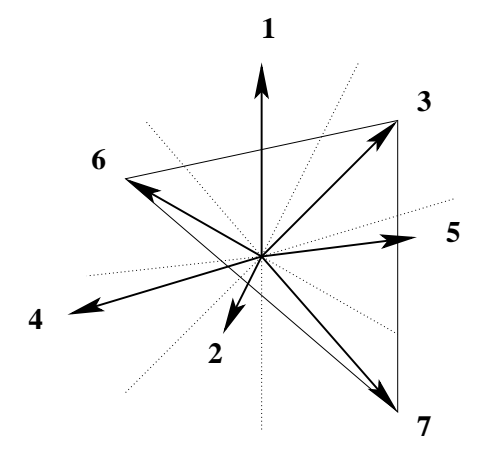

Figure 5: A Gale transform of $C_{4}(7)$. The set $N_{\boldsymbol{p}}=\{3,6,7\}$ corresponds to the vertex $\boldsymbol{p}$ of $C_{4}(7)^{\Delta}$ not on those facets, and $3<6<7$ is an anti-clockwise orientation of the triangle 367 .

Lemma 5.4. Let $\mathcal{G}(P)$ be an extended Gale diagram of $P=C_{d}(d+3)^{\Delta}$, and let $N_{\boldsymbol{p}}=$ $\{i, j, k\}$ index a vertex $\boldsymbol{p}$ of $P$. With the assumptions of Observation 3.2 and Convention 5.1, the intersection height $z_{\boldsymbol{p}}=z_{\{i, j, k\}}$ is given by

$$
z_{\{i, j, k\}}=\frac{\boldsymbol{i} \boldsymbol{j}^{\perp} h_{k}+\boldsymbol{k} \boldsymbol{i}^{\perp} h_{j}+\boldsymbol{j}^{\perp} h_{i}}{[\boldsymbol{i} \boldsymbol{j} \boldsymbol{k}]} .
$$

Proof. Expand the third row of the determinant in the equation

$$
\left|\begin{array}{cccc}
0 & x_{i} & x_{j} & x_{k} \\
0 & y_{i} & y_{j} & y_{k} \\
z_{\{i, j, k\}} & h_{i} & h_{j} & h_{k} \\
1 & 1 & 1 & 1
\end{array}\right|=0 .
$$

As a consistency check, note that (10) is symmetric under any permutation of the indices. $\square$

By Proposition 3.3. the total ordering of the vertices $\boldsymbol{p}$ of $C_{d}(d+3)^{\Delta}$ induced by the linear objective function $\boldsymbol{g}^{T}$ induces a total ordering of the intersection heights $z_{\boldsymbol{p}}$, that is, of the heights of the intersections of the affine hyperplanes $H_{\boldsymbol{p}}$ in $\mathbb{R}^{3}$ with the $z$-axis. If two vertices of $C_{d}(d+3)^{\Delta}$ span an edge, then the corresponding facets of $C_{d}(d+3)$ share a ridge, which in turn means that the corresponding triangles have two points $\boldsymbol{w}_{i}^{*}, \boldsymbol{w}_{j}^{*}$ in common. This permits us to relate the intersection heights of two adjacent vertices in the graph of $C_{d}(d+3)^{\Delta}$ in the following way.

Lemma 5.5. Suppose that the vertices $\{i, j, k\}$ and $\{i, j, \ell\}$ span an edge of $C_{d}(d+3)^{\Delta}$. Then the following relation holds between the corresponding intersection heights:

$$
z_{\{i, j, k\}}-z_{\{i, j, \ell\}}=\frac{\left(\boldsymbol{i} \boldsymbol{j}^{\perp}\right)[\boldsymbol{j} \boldsymbol{k} \boldsymbol{\ell}]}{[\boldsymbol{i} \boldsymbol{j} \boldsymbol{k}][\boldsymbol{i} \boldsymbol{j} \ell]} h_{i}+\frac{\left(\boldsymbol{i} \boldsymbol{j}^{\perp}\right)[\boldsymbol{k} \boldsymbol{i} \boldsymbol{\ell}]}{[\boldsymbol{i} \boldsymbol{j} \boldsymbol{k}][\boldsymbol{i} \boldsymbol{j} \ell]} h_{j}+\frac{\boldsymbol{i} \boldsymbol{j}^{\perp}}{[\boldsymbol{i} \boldsymbol{j} \boldsymbol{k}]} h_{k}+\frac{-\boldsymbol{i} \boldsymbol{j}^{\perp}}{[\boldsymbol{i} \boldsymbol{j} \ell]} h_{\ell} .
$$

If $[i j k]=[i j \ell]$, then the signs of the coefficients of the $h$ 's are, in this order,

$$
\left(i j^{\perp}\right)[j k \ell], \quad\left(i j^{\perp}\right)[k i \ell], \quad+, \quad-.
$$




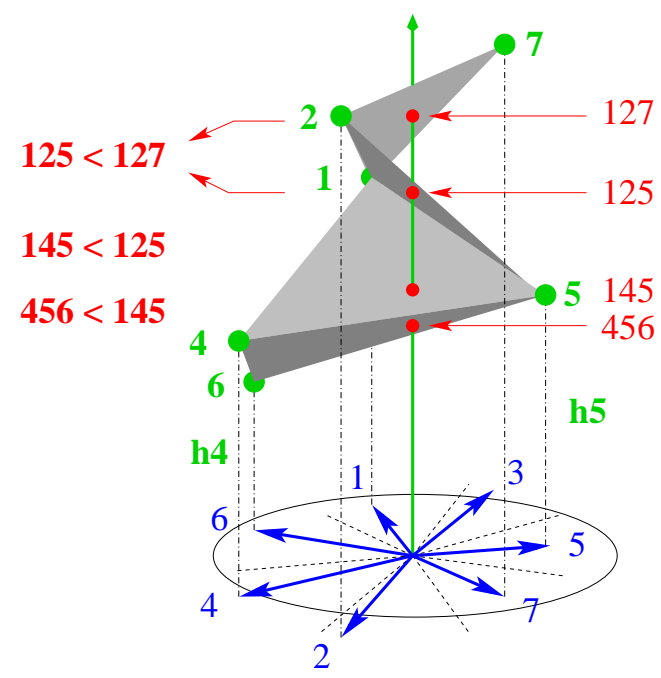

FigURE 6: Intersection heights encode values of the objective function. Suppose that the objective function $\tilde{g}$ orders four vertices of $C_{4}(7)$ by $1237<2367<3467<3456$. Then the heights of the intersections between $\mathbb{R} \tilde{g}^{*}$ and the lifted triangles corresponding to the complements of these labels are ordered $z_{456}<z_{145}<z_{125}<z_{127}$.

Proof. The first statement follows via direct calculation from (1), using the straightforward identity

$$
\left(\boldsymbol{i} \boldsymbol{j}^{\perp}\right)\left(\boldsymbol{k} \ell^{\perp}\right)=\left(\boldsymbol{\ell} \boldsymbol{i}^{\perp}\right)\left(\boldsymbol{j} \boldsymbol{k}^{\perp}\right)+\left(\boldsymbol{j} \ell^{\perp}\right)\left(\boldsymbol{i} \boldsymbol{k}^{\perp}\right) .
$$

The second statement is a consequence of Lemma 5.2 and Convention 5.3

\subsection{Contradictions via a combinatorial Farkas Lemma}

We will use a combinatorial version of the following Farkas Lemma [18, Sect. 7.8]:

Lemma 5.6. For any matrix $A \in \mathbb{R}^{m \times d}$, exactly one of the following is true:

- There exists an $\boldsymbol{h} \in \mathbb{R}^{d}$ such that $A \boldsymbol{h}<\mathbf{0}$.

- There exists a $\boldsymbol{c} \in \mathbb{R}^{m}$ such that $\boldsymbol{c} \geq \mathbf{0}, \boldsymbol{c}^{T} A=\mathbf{0}$, and $\boldsymbol{c} \neq \mathbf{0}$.

Given a $d$-dimensional polytope $P$ with $d+3$ facets and an orientation $\mathcal{O}$ on $P$ 's graph $G$, we assume that we have a realization of $P$ and a linear objective function $\boldsymbol{g}^{T}$ that induces $\mathcal{O}$ on $G$. We would like to apply Lemma 5.6 to prove the infeasibility of the system $A \boldsymbol{h}<\mathbf{0}$ of $m=$ \# edges of $C_{d}(d+3)^{\Delta}=\frac{1}{4}\left(\begin{array}{c}d+4 \\ 3\end{array}\right)$ linear inequalities on the lifting heights $h_{1}, h_{2}, \ldots, h_{d+3}$ given by

$$
z_{\{i, j, k\}}-z_{\{i, j, \ell\}}<0 \quad \text { for all oriented edges }(\{i, j, k\},\{i, j, \ell\}) \text { of } \mathcal{O} .
$$

However, the only information we have available about $A$ are sign patterns of determinants as given by Lemma 5.5. Therefore, to show infeasibility of (2) we must produce a Farkas certificate $\boldsymbol{c}$ that shows already at the level of signs ("using only oriented matroid information") that some positive combination of the rows of $A$ sums to zero. 
Proposition 5.7. The orientation

$$
\begin{aligned}
N R_{1}^{4}: \quad z_{145} & <z_{147}<z_{127}<z_{125}<z_{123}<z_{236}<z_{234}< \\
& <z_{345}<z_{347}<z_{367}<z_{167}<z_{567}<z_{256}<z_{456}
\end{aligned}
$$

of the graph of $C_{4}(7)^{\Delta}$ is not realizable.

Proof. We abbreviate ' $z_{\{i, j, k\}}<z_{\{i, j, \ell\}}$ ' by 'ijk $<i j \ell$ '.

To any extended Gale diagram corresponding to a realization of $N R_{1}^{4}$ we may apply an affine transformation that fixes the $z$-axis and moves the plane spanned by $\boldsymbol{w}_{3}^{*}, \boldsymbol{w}_{4}^{*}$ and $\boldsymbol{w}_{5}^{*}$ to the $\mathbb{R}^{2}$-plane given by $z=0$; that is, we may assume that $h_{3}=h_{4}=h_{5}=0$. This affine transformation does not change the projection along the $z$-axis, which still yields the same Gale transform of $C_{4}(7)$. The resulting configuration is the extended Gale diagram for $C_{4}(7)^{\Delta}$ with the objective function $\tilde{\boldsymbol{g}}=c \boldsymbol{g}$ scaled such that the level hyperplane $\tilde{\boldsymbol{g}}^{T} \boldsymbol{x}=1$ contains $\boldsymbol{p}=\{3,4,5\}$. Thus at this point we have modified Step (1) in the construction of Section 3 .

We proceed to write down the sign patterns of the inequalities $A \boldsymbol{h}<\mathbf{0}$ for $\boldsymbol{h}=$ $\left(h_{1}, h_{2}, h_{6}, h_{7}\right)$ implied by Lemmas 5.4 and 5.5

\begin{tabular}{c|cccc|cc} 
& $h_{1}$ & $h_{2}$ & $h_{6}$ & $h_{7}$ & $i j k \ell$ \\
\hline $567<256:$ & 0 & - & $-[257]$ & + & 5672 \\
$234<345:$ & 0 & + & 0 & 0 & 34425 \\
$345<456:$ & 0 & 0 & - & 0 & 4536 \\
$345<347:$ & 0 & 0 & 0 & - & 3457
\end{tabular}

If $[257]=-$ or $[257]=0$, we can find a positive combination of the rows of this matrix that sums to zero, regardless of the actual values of the entries. Therefore, $[257]=+$ in any realization of $N R_{1}^{4}$. By Lemma [5.2(D), we deduce that therefore $[157]=-$.

\begin{tabular}{|c|c|c|c|c|c|}
\hline & $h_{1}$ & $h_{2}$ & $h_{6}$ & $h_{7}$ & $i j k \ell$ \\
\hline $127<125:$ & $-[257]=-$ & {$[157]=-$} & 0 & + & 1275 \\
\hline $145<345$ : & + & 0 & 0 & 0 & 4513 \\
\hline $234<345:$ & 0 & + & 0 & 0 & 3425 \\
\hline $345<347$ : & 0 & 0 & 0 & - & 3457 \\
\hline
\end{tabular}

Now consider the rows

which admit a positive combination that sums to zero and therefore prove the nonrealizability of the orientation $N R_{1}^{4}$.

Remark. Proposition 5.7provides an example of a non-realizable abstract objective function that satisfies the Holt-Klee conditions, on a simple 4-polytope with only 7 facets. The first examples for this were obtained on a 7-dimensional polytope with 9 facets, by Gärtner et al. 3]; Morris [15] obtained examples on the 4-cube (with 8 facets). No such examples of dimension $d \leq 3$ exist (Mihalisin \& Klee [14]).

Proposition 5.8. No Hamilton $H K$ AOF of $C_{6}(9)^{\Delta}$ is realizable.

Proof. The reasoning is analogous to the proof of Proposition 5.7 we will give the details only for $N R_{1}^{6}$, and sketch the proof for the rest of the orientations. 
Suppose then that we are given a realization of the polytope $C_{6}(9)^{\Delta}$ along with a linear objective function that induces $N R_{1}^{6}$ on its graph. After an affine transformation of the extended Gale diagram, we may suppose that $h_{3}=h_{4}=h_{5}=0$, where we consider the lifting heights numbered as $h_{0}, h_{1}, \ldots, h_{8}$.

Now consider the rows

\begin{tabular}{|c|c|c|c|c|c|c|c|}
\hline & $h_{0}$ & $h_{1}$ & $h_{2}$ & $h_{6}$ & $h_{7}$ & $h_{8}$ & $i j k \ell$ \\
\hline $567<056$ & - & 0 & 0 & $-[057]$ & + & 0 & 5670 \\
\hline $034<345$ & + & 0 & 0 & 0 & 0 & 0 & 405 \\
\hline $345<456$ & 0 & 0 & 0 & - & 0 & 0 & 4536 \\
\hline $345<347$ & 0 & 0 & 0 & 0 & - & 0 & 3457 \\
\hline
\end{tabular}

from which we deduce as above that $[057]=+$, and via Lemma $5.2(\mathrm{~b})$ that $[578]=-$. But now we reach a contradiction via

\begin{tabular}{|c|c|c|c|c|c|c|c|}
\hline & $h_{0}$ & $h_{1}$ & $h_{2}$ & $h_{6}$ & $h_{7}$ & $h_{8}$ & $i j k \ell$ \\
\hline $078<058:$ & {$[578]=-$} & 0 & 0 & 0 & + & $-[057]=-$ & 0875 \\
\hline $034<345:$ & + & 0 & 0 & 0 & 0 & 0 & 3405 \\
\hline $345<347$ : & 0 & 0 & 0 & 0 & - & 0 & 3457 \\
\hline $458<345:$ & 0 & 0 & 0 & 0 & 0 & + & 4583 \\
\hline
\end{tabular}

which proves the claim. Some "good" sets of vanishing heights for the remaining orientations are as follows:

\begin{tabular}{l|ccccc} 
Orientation: & $N R_{2}^{6}$ & $N R_{3}^{6}$ & $N R_{4}^{6}$ & $N R_{5}^{6}$ & $N R_{6}^{6}$ \\
\hline Height indices: & $0,1,2$ & $0,5,6$ & $0,5,6$ & $0,1,6$ & $0,1,2$
\end{tabular}

This concludes the proof.

Proposition 5.9. The Hamilton HK AOFs $N R_{2}^{4}$ and $N R_{3}^{4}$ are not realizable.

Proof. The method used in the proof of Propositions 5.7 and 5.8 does not directly apply here, as no choice of vanishing heights immediately yields a Farkas contradiction for these orientations. Therefore we prove the nonrealizability of $N R_{2}^{4}$ in the following way:

Suppose that in a realization of $N R_{2}^{4}$, we have [136] $=+$, and therefore [137] $=-$ by Lemma $5.2(\mathrm{~b})$. This leads to a contradiction by the following table for $h_{1}=h_{4}=h_{5}=0$ :

\begin{tabular}{c|cccc} 
& $h_{2}$ & $h_{3}$ & $h_{6}$ & $h_{7}$ \\
\hline $367<167:$ & 0 & + & $-[137]=+$ & {$[136]=+$} \\
$145<345:$ & 0 & - & 0 & 0 \\
$145<456:$ & 0 & 0 & - & 0 \\
$145<147:$ & 0 & 0 & 0 & -
\end{tabular}

We deduce that $[136]=-$ or $[136]=0$ must hold in any realization of $N R_{2}^{4}$. But setting $h_{2}=h_{3}=h_{6}=0$ then yields the following table,

\begin{tabular}{c|cccc} 
& $h_{1}$ & $h_{4}$ & $h_{5}$ & $h_{7}$ \\
\hline $367<167:$ & - & 0 & 0 & {$[136]$} \\
$123<236:$ & + & 0 & 0 & 0 \\
$367<236:$ & 0 & 0 & 0 & +
\end{tabular},


and a global contradiction.

The same argument proves that $N R_{3}^{4}$ is nonrealizable. The only difference between this orientation and $N R_{2}^{4}$ is that $345<347$ in $N R_{3}^{4}$, whereas $347<345$ in $N R_{2}^{4}$, but the proof of the nonrealizability of $N R_{2}^{4}$ did not use this inequality.

Remark. The short non-realizability proofs above were found by computer, though they can be checked by hand. Propositions 5.7 and 5.8 were found by trying to eliminate signs from all minors of $A$ obtained by successively deleting triples of columns, while the proof of Proposition [5.9] was obtained by moreover assuming various signs to be positive resp. negative. We presented instances of the shortest proofs found.

\section{Summary and open problems}

Our strategy for proving bounds on $M(d, n)$ depends on combining theoretical concepts with computer generation/enumeration. For lower bounds on $M(d, n)$, we use

(1) information on the combinatorial type of a candidate polytope - e.g., the combinatorial type of a 6 -dimensional 9-facet polytope with maximally many vertices is uniquely that of $C_{6}(9)^{\Delta}$;

(2) reduction of the dimension via extended Gale diagrams - to dimension 3 in the case of $C_{6}(9)^{\Delta}$

(3) random generation of instances of the extended Gale diagram, and finding the longest ascending path on the induced orientation (this step involves the computer).

Our proof of the upper bound $M(6,9)<30$ additionally uses

(4) the Holt-Klee and AOF properties of orientations induced by linear functions;

(5) exhaustive enumeration (up to symmetry) of all orientations that admit a Hamilton path and satisfy the HK and AOF conditions;

(6) a combinatorial version of the Farkas Lemma;

(7) computer assistance to find short nonrealizability proofs.

Step (5) of our present proof depends crucially on computer assistance. Finding short proofs in step (17) also requires computer assistance, but the result can be checked by hand.

Our methods were successful for small dimensions and coranks, but they do not yield (non-)existence statements or asymptotics for large $d$ and $n-d$. Thus we leave the following problems open for now:

- Does $C_{d}(d+3)^{\Delta}$ have any Hamilton HK AOFs for even $d>6$ ? If not, this would give a purely combinatorial proof that for some parameters $M(d, n)<M_{\mathrm{ubt}}(d, n)$.

What happens for odd $d \geq 7$ ?

- Is it true that $M(d, n) \ll M_{\mathrm{ubt}}(d, n)$ for large $n \geq d+3, d \geq 6$ ?

To demonstrate that the gaps in our asymptotic knowledge are substantial, we note that in the "diagonal" case of $n=2 d$ all we know is

$$
\frac{5}{4} 2^{d} \leq M(d, 2 d) \leq M_{\mathrm{ubt}}(d, 2 d) \approx 2.6^{d} .
$$

(The lower bound follows from $M(4,8)=20$ by taking deformed prisms.) 


\section{Acknowledgements}

The first author is grateful for an initiation to the power of the extended Gale diagram by Emo Welzl himself, during a stay at ETH Zürich, and wishes to thank Walter Morris for many discussions. Volker Kaibel has contributed to this paper through numerous discussions and useful observations, and Christoph Eyrich through uncommon typographical expertise.

\section{References}

[1] A. Altshuler And P. McMullen, The number of simplicial neighborly d-polytopes with $d+3$ vertices, Mathematika, 20 (1973), pp. 263-266.

[2] N. Amenta And G. M. Ziegler, Deformed products and maximal shadows, in Advances in Discrete and Computational Geometry (South Hadley, MA, 1996), B. Chazelle, J. E. Goodman, and R. Pollack, eds., vol. 223 of Contemporary Mathematics, Amer. Math. Soc., Providence RI, 1998, pp. 57-90.

[3] B. Gärtner, J. Solymosi, F. Tschirschnitz, P. Valtr, and E. Welzl, One line and $n$ points, in Proc. 33rd Ann. ACM Symp. on the Theory of Computing (STOC), 2001, pp. 306-315.

[4] E. Gawrilow And M. Joswig, Polymake: A software package for analyzing convex polytopes. http://www.math.tu-berlin.de/diskregeom/polymake/.

[5] —, Polymake: A framework for analyzing convex polytopes, in Polytopes - Combinatorics and Computation, G. Kalai and G. M. Ziegler, eds., vol. 29 of DMV Seminar, Birkhäuser-Verlag, Basel, 2000, pp. 43-73.

[6] B. Grünbaum, Convex Polytopes, vol. 221 of Graduate Texts in Math., SpringerVerlag, New York, 2003. Second edition edited by V. Kaibel, V. Klee and G. M. Ziegler (original edition: Interscience, London 1967).

[7] C. HaAse And G. M. Ziegler, Examples and counterexamples for the Perles conjecture, Discrete Comput. Geometry, 28 (2002), pp. 29-44. DOI: 10.1007/s00454-0010085-0; math.C0/0011170; MR 2003e:52016, Zbl 1011.52005.

[8] F. Holt And V. KleE, A proof of the strict monotone 4-step conjecture, in Advances in Discrete and Computational Geometry (South Hadley, MA, 1996), B. Chazelle, J. E. Goodman, and R. Pollack, eds., vol. 223 of Contemporary Mathematics, Providence RI, 1998, Amer. Math. Soc., pp. 201-216.

[9] M. Joswig, V. KAIBel, And F. KÖRner, On the $k$-systems of a simple polytope, Israel J. Math., 129 (2002), pp. 109-118. 
[10] G. Kalai, A simple way to tell a simple polytope from its graph, J. Combinatorial Theory, Ser. A, 49 (1988), pp. 381-383.

[11] V. KleE, Paths on polyhedra I, J. Soc. Indust. Appl. Math., 13 (1965), pp. 946-956.

[12] V. Klee And G. J. Minty, How good is the simplex algorithm?, in Inequalitites, III, O. Shisha, ed., Academic Press, New York, 1972, pp. 159-175.

[13] P. MCMullen, The numbers of faces of simplicial polytopes, Israel J. Math., 9 (1971), pp. 559-570.

[14] J. Mihalisin And V. Klee, Convex and linear orientations of polytopal graphs, Discrete Comput. Geometry, 24 (2000), pp. 421-436. The Branko Grünbaum birthday issue (G. Kalai, V. Klee, eds.).

[15] W. D. MorRIs, Distinguishing cube orientations arising from linear programs. Preprint, 12 pages, September 2002.

[16] T. S. Motzkin, Comonotone curves and polyhedra, Bulletin Amer. Math. Soc., 63 (1957), p. 35. Abstract.

[17] J. Pfeifle, Extremal Constructions for Polytopes and Spheres, PhD thesis, Technische Universität Berlin, 2003.

[18] A. SchriJver, Theory of Linear and Integer Programming, Wiley-Interscience Series in Discrete Mathematics and Optimization, John Wiley and Sons, Chichester NY, 1986. Reprint 1998.

[19] E. WelzL, Entering and leaving j-facets, Discrete Comput. Geometry, 25 (2001), pp. 351-364.

[20] G. M. Ziegler, Lectures on Polytopes, vol. 152 of Graduate Texts in Mathematics, Springer-Verlag, New York, revised ed., 1998. Revised edition, 1998; "Updates, corrections, and more" at Www.math.tu-berlin.de/ ${ }^{\sim}$ ziegler.

[21] — Questions about polytopes, in Mathematics Unlimited - 2001 and Beyond, B. Enquist and W. Schmid, eds., Springer-Verlag, Berlin Heidelberg, 2001, pp. 1195-1211. 